Louisiana State University

LSU Digital Commons

Faculty Publications

Department of Physics \& Astronomy

$11-1-2007$

\title{
Results of the IGEC-2 search for gravitational wave bursts during 2005
}

\author{
P. Astone \\ Istituto Nazionale di Fisica Nucleare - INFN \\ D. Babusci \\ INFN, Laboratori Nazionali Di Frascati \\ L. Baggio \\ European Gravitational Observatory (EGO) \\ M. Bassan \\ Università degli Studi di Roma "Tor Vergata" \\ M. Bignotto \\ Università degli Studi di Padova
}

See next page for additional authors

Follow this and additional works at: https://digitalcommons.Isu.edu/physics_astronomy_pubs

\section{Recommended Citation}

Astone, P., Babusci, D., Baggio, L., Bassan, M., Bignotto, M., Bonaldi, M., Camarda, M., Carelli, P., Cavallari, G., Cerdonio, M., Chincarini, A., Coccia, E., Conti, L., D'Antonio, S., De Rosa, M., Di Paolo Emilio, M., Drago, M., Dubath, F., Fafone, V., Falferi, P., Foffa, S., Fortini, P., Frasca, S., Gemme, G., Giordano, G., Giusfredi, G., Hamilton, W., Hanson, J., Inguscio, M., Johnson, W., Liguori, N., Longo, S., \& Maggiore, M. (2007). Results of the IGEC-2 search for gravitational wave bursts during 2005. Physical Review D - Particles, Fields, Gravitation and Cosmology, 76 (10) https://doi.org/10.1103/PhysRevD.76.102001

This Article is brought to you for free and open access by the Department of Physics \& Astronomy at LSU Digital Commons. It has been accepted for inclusion in Faculty Publications by an authorized administrator of LSU Digital Commons. For more information, please contact ir@lsu.edu. 


\section{Authors}

P. Astone, D. Babusci, L. Baggio, M. Bassan, M. Bignotto, M. Bonaldi, M. Camarda, P. Carelli, G. Cavallari, M. Cerdonio, A. Chincarini, E. Coccia, L. Conti, S. D'Antonio, M. De Rosa, M. Di Paolo Emilio, M. Drago, F. Dubath, V. Fafone, P. Falferi, S. Foffa, P. Fortini, S. Frasca, G. Gemme, G. Giordano, G. Giusfredi, W. O.

Hamilton, J. Hanson, M. Inguscio, W. W. Johnson, N. Liguori, S. Longo, and M. Maggiore 


\title{
Results of the IGEC-2 search for gravitational wave bursts during 2005
}

P. Astone, ${ }^{1}$ D. Babusci, ${ }^{2}$ L. Baggio, ${ }^{3}$ M. Bassan,${ }^{4,5}$ M. Bignotto, ${ }^{6,7}$ M. Bonaldi, ${ }^{8,9}$ M. Camarda, ${ }^{10}$ P. Carelli,,${ }^{5,11}$ G. Cavallari, ${ }^{12}$ M. Cerdonio, ${ }^{6,7}$ A. Chincarini, ${ }^{13}$ E. Coccia,${ }^{4,14}$ L. Conti, ${ }^{6,7}$ S. D’ Antonio, ${ }^{5}$ M. De Rosa, ${ }^{15,16}$ M. di Paolo Emilio, ${ }^{11,14}$ M. Drago,${ }^{6,7}$ F. Dubath, ${ }^{17}$ V. Fafone,${ }^{4,5}$ P. Falferi, ${ }^{8,9}$ S. Foffa, ${ }^{17}$ P. Fortini, ${ }^{18}$ S. Frasca, ${ }^{1,19}$ G. Gemme ${ }^{13}$ G. Giordano, ${ }^{2}$ G. Giusfredi,${ }^{20}$ W. O. Hamilton, ${ }^{21}$ J. Hanson, ${ }^{21}$ M. Inguscio, ${ }^{16,22}$ W. W. Johnson, ${ }^{21}$ N. Liguori, ${ }^{6,7}$ S. Longo, ${ }^{23}$ M. Maggiore, ${ }^{17}$ F. Marin, ${ }^{16,22}$ A. Marini, ${ }^{2}$ M. P. McHugh, ${ }^{24}$ R. Mezzena, ${ }^{9,25}$ P. Miller, ${ }^{21}$ Y. Minenkov, ${ }^{14}$ A. Mion, ${ }^{9,25}$ G. Modestino, ${ }^{2}$ A. Moleti, ${ }^{4,5}$ D. Nettles, ${ }^{21}$ A. Ortolan, ${ }^{23}$ G. V. Pallottino, ${ }^{1,19}$ R. Parodi, ${ }^{13}$ G. Piano Mortari, ${ }^{11,14}$ S. Poggi, ${ }^{26}$ G. A. Prodi,${ }^{9,25, *}$ L. Quintieri, ${ }^{2}$ V. Re, ${ }^{9,25}$ A. Rocchi, ${ }^{4}$ F. Ronga, ${ }^{2}$ F. Salemi,,${ }^{9,25}$ G. Soranzo, ${ }^{7}$ R. Sturani, ${ }^{17}$ L. Taffarello, ${ }^{7}$ R. Terenzi, ${ }^{1,27}$ G. Torrioli, ${ }^{1,28}$ R. Vaccarone, ${ }^{13}$ G. Vandoni, ${ }^{12}$ G. Vedovato, ${ }^{7}$ A. Vinante,${ }^{8,9}$ M. Visco, ${ }^{4,27}$ S. Vitale, ${ }^{9,25}$ J. Weaver,${ }^{21}$ J. P. Zendri, ${ }^{7}$ and P. Zhang ${ }^{21}$

(IGEC-2 Collaboration)

\author{
${ }^{1}$ INFN, Sezione di Roma, Piazzale le A. Moro 2, I-00185, Roma, Italy \\ ${ }^{2}$ INFN, Laboratori Nazionali di Frascati, Via E. Fermi 40, I-00044, Frascati, Italy \\ ${ }^{3}$ EGO, 56021 Santo Stefano a Macerata, Cascina, Pisa, Italy \\ ${ }^{4}$ Dipartimento di Fisica, Università di Roma “Tor Vergata," Via Ricerca Scientifica 1, I-00133 Roma, Italy \\ ${ }^{5}$ INFN, Sezione di Roma Tor Vergata, Via Ricerca Scientifica 1, I-00133 Roma, Italy \\ ${ }^{6}$ Dipartimento di Fisica, Università di Padova, Via Marzolo 8, 35131 Padova, Italy \\ ${ }^{7}$ INFN, Sezione di Padova, Via Marzolo 8, 35131 Padova, Italy \\ ${ }^{8}$ Istituto di Fotonica e Nanotecnologie, CNR-Fondazione Bruno Kessler, I-38050 Povo, Trento, Italy \\ ${ }^{9}$ INFN, Gruppo Collegato di Trento, Sezione di Padova, I-38050 Povo, Trento, Italy \\ ${ }^{10}$ Dipartimento di Ingegneria Informatica, Università di Padova, Via G. Gradenigo 6a, 35131 Padova, Italy \\ ${ }^{11}$ Dipartimento di Fisica, Università de L'Aquila, L'Aquila, Italy \\ ${ }^{12}$ CERN, Geneva, Switzerland \\ ${ }^{13}$ INFN, Sezione di Genova, Genova, Italy \\ ${ }^{14}$ INFN, Laboratori Nazionali del Gran Sasso, Assergi, L’Aquila, Italy \\ ${ }^{15}$ INOA, CNR, I-80078 Pozzuoli, Napoli, Italy \\ ${ }^{16}$ INFN, Sezione di Firenze, I-50121 Firenze, Italy \\ ${ }^{17}$ Département de Physique Théorique, Université de Genève, Genève, Switzerland \\ ${ }^{18}$ Dipartimento di Fisica, Università di Ferrara and INFN, Sezione di Ferrara, I-44100 Ferrara, Italy \\ ${ }^{19}$ Dipartimento di Fisica, Università di Roma "La Sapienza," Piazzale le A. Moro 2, I-00185, Roma, Italy \\ ${ }^{20}$ INOA, CNR, I-50125 Arcetri, Firenze, Italy \\ ${ }^{21}$ Department of Physics and Astronomy, Louisiana State University, Baton Rouge, Louisiana 70803, USA \\ ${ }^{22}$ LENS and Dipartimento di Fisica, Università di Firenze, I-50121 Firenze, Italy \\ ${ }^{23}$ INFN, Laboratori Nazionali di Legnaro, 35020 Legnaro, Padova, Italy \\ ${ }^{24}$ Department of Physics, Loyola University New Orleans, New Orleans, Louisiana 70118, USA \\ ${ }^{25}$ Dipartimento di Fisica, Università di Trento, I-38050 Povo, Trento, Italy \\ ${ }^{26}$ Consorzio Criospazio Ricerche, I-38050 Povo, Trento, Italy \\ ${ }^{27}$ INAF, Istituto Fisica Spazio Interplanetario,Via Fosso del Cavaliere, I-00133 Roma, Italy \\ ${ }^{28}$ CNR, Istituto di Fotonica e Nanotecnologie, Roma, Italy \\ (Received 11 June 2007; published 1 November 2007)
}

The network of resonant bar detectors of gravitational waves resumed coordinated observations within the International Gravitational Event Collaboration (IGEC-2). Four detectors are taking part in this Collaboration: ALLEGRO, AURIGA, EXPLORER and NAUTILUS. We present here the results of the search for gravitational wave bursts over 6 months during 2005, when IGEC-2 was the only gravitational wave observatory in operation. The implemented network data analysis is based on a time coincidence search among AURIGA, EXPLORER and NAUTILUS; ALLEGRO data was reserved for follow-up studies. The network amplitude sensitivity to bursts improved by a factor $\approx 3$ over the 1997-2000 IGEC observations; the wider sensitive band also allowed the analysis to be tuned over a larger class of waveforms. Given the higher single-detector duty factors, the analysis was based on threefold coincidence, to ensure the identification of any single candidate of gravitational waves with high statistical confidence. The false detection rate was as low as 1 per century. No candidates were found.

\footnotetext{
*Corresponding author. prodi@science.unitn.it
} 


\section{INTRODUCTION}

The search for unmodeled, transient gravitational waves (GWs) demands the use of a network of detectors. The joint analysis of data from multiple detectors at different locations allows one to reject efficiently spurious candidates caused by transient local disturbances or by intrinsic detector noise. Moreover, the false alarm probability of the network due to uncorrelated noise sources at the different sites can be reliably estimated.

The first long term search for bursts GW by a network of detectors was performed by the five resonant bars ALLEGRO, AURIGA, EXPLORER, NAUTILUS and NIOBE, within the International Gravitational Event Collaboration (IGEC) [1]. The search consisted in a time coincidence analysis over a 4-year period, from 1997 to 2000. It set an upper limit on the rate of impulsive gravitational waves as a function of the GW amplitude threshold of the data analysis [2]. The overlap in observation time of the detectors was modest: three or more detectors were in simultaneous validated observation for 173 days, $\simeq 12 \%$ of the total time, and twofold observations covered an additional period of 534 days, or $\simeq 36 \%$ of the total time. Moreover, since some false alarms were to be expected in the twofold coincidence analysis at the lowest amplitude thresholds, most of the time the IGEC 1997-2000 observation was not able to discriminate a single $\mathrm{GW}$ candidate from accidental coincidences. The target GW signals were short transients showing a flat Fourier spectrum around $900 \mathrm{~Hz}$, such as pulses of $\sim 1 \mathrm{~ms}$ duration or oscillating signals with a few cycles of $\sim 1$ ms period.

The same class of signals was targeted by searches on EXPLORER and NAUTILUS data in 2001 [3] and 2003 [4]. These searches, based on twofold coincidences, could not identify single GW candidates, but they studied a possible excess of coincidences through a sidereal time analysis.

Networks of interferometric detectors performed more recent searches, with better sensitivity over a wider frequency band. In particular, the sensitivity of LIGO burst searches significantly improved from the S2 run in 2003 [5] to the S4 run in 2005 [6]. The upper limit set by IGEC on the rate of millisecond GW signals continues to be competitive at large GW amplitude, due to the short live time of the interferometer analyses. However, this may soon change, since in November 2005 the LIGO observatory started the S5 run, its first long term data acquisition at design sensitivity [7].

In 2004 four resonant bar detectors resumed simultaneous operation after a round of upgrades: ALLEGRO [8], AURIGA [9,10], EXPLORER and NAUTILUS [11,12]. A new GW search was initiated by the IGEC-2 Collaboration, with the goal to identify GW burst candidates with high statistical confidence. This coordinated observa- tion is still running and targets to a broader signal class than the previous IGEC search, including such sources as binary black hole mergers and ring-downs [13] and the longer transients recently predicted for Supernova core collapses [14].

This paper is the first report on the IGEC-2 observations and describes the results of the analysis of 6 months of data, from 20 May to 15 November 2005, when IGEC-2 was the only gravitational wave observatory in operation. The AURIGA, EXPLORER and NAUTILUS data are actually used to search for gravitational wave candidates showing up as triple time coincidences. Because of a delay in the validation of the ALLEGRO data, we agreed to use the data from this detector for follow-up investigations on possible signal candidates identified by the other resonant bars.

\section{CHARACTERISTICS AND GOALS OF THE IGEC-2 OBSERVATORY}

As for the previous IGEC search in 1997-2000, the detectors are aligned within a few degrees and so feature the same directional sensitivity at any time. The spectral sensitivities of the resonant bar detectors during 2005 is shown in Fig. 1. The minima of the noise power spectral densities are very close, within $1 \div 2 \times 10^{-21} 1 / \sqrt{\mathrm{Hz}}$, as the four detectors share a similar design (i.e. cylindrical Al5056 bar with a mass of $\simeq 2200 \mathrm{~kg}$ cooled at liquid $\mathrm{He}$ temperature, resonant transducers, similar mechanical quality factors, DC-SQUID signal amplifier). The detectors exhibit improved performance and wider bandwidth than the original IGEC network [2]. EXPLORER and NAUTILUS were upgraded in 2000 and 2002, respectively,

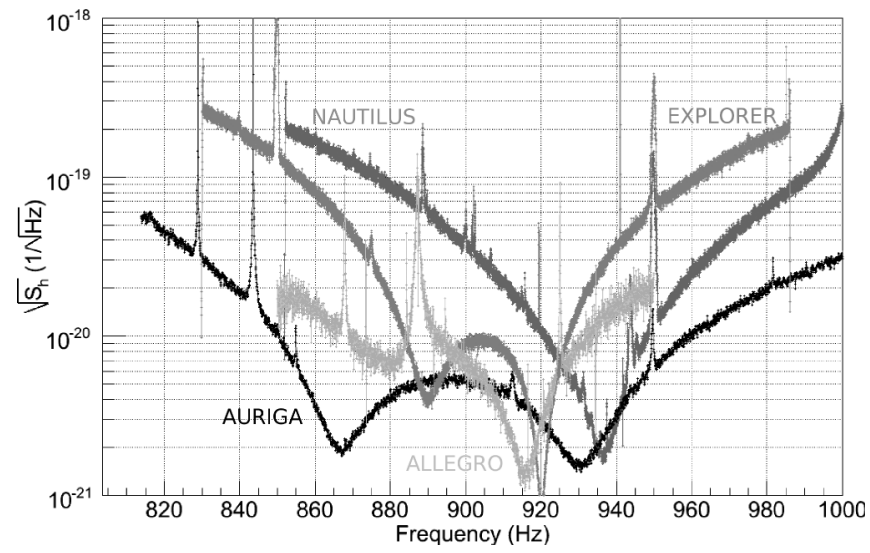

FIG. 1. Typical strain noise spectral density (single-sided) curves of IGEC-2 detectors in 2005. From light gray to black: ALLEGRO, EXPLORER, NAUTILUS, and AURIGA. All detectors share comparable minimum levels of noise spectra. The wider bandwidth of AURIGA covers the bandwidths of the other detectors. 
with some modifications on the cryogenic apparatus and mechanical filters, and the adoption of new transducers and new DC-SQUIDs [15]. The upgrade of AURIGA, completed in 2003, affected most of the apparatus, from the seismic isolation system [16] to the readout [17]. In particular, a better coupling between transducer and signal amplifier was achieved by tuning the electric resonance of the signal transformer to the mechanical modes of the antenna and transducer; the signal amplifier is now based on a two stage DC-SQUID. The result was a very large increase in the detector bandwidth [10,18]. Additional upgrades of the room temperature suspensions during 2005 led to a significant improvement in duty-cycle and data quality. ALLEGRO resumed operation in early 2004, after changing both the resonant transducer and the readout electronics [8].

The primary scientific goal of the IGEC-2 observations is to identify single gravitational wave candidates with high statistical confidence.

\section{CHARACTERISTICS OF THE EXCHANGED DATA}

During the 180 days considered in this analysis, the AURIGA, EXPLORER and NAUTILUS detectors show a high duty cycle, see Table I. In particular the validated data of AURIGA, EXPLORER and NAUTILUS overlap by 130.71 days in threefold coincidence (corresponding to $73 \%$ ) and 45 days more are covered as twofold coincidences (about 25\%).

The detectors noises are remarkably stable, especially if compared to past performances. As shown in Fig. 2, the standard deviation of AURIGA noise shows a slow systematic dependence on the liquid He levels in the cryostats with peak-to-peak variations of the order of $10 \%$. Minimum noise levels of EXPLORER and NAUTILUS are higher by a factor of $\sim 2$ in terms of equivalent amplitude of a millisecond gravitational wave burst.

Each group independently validates its data and tunes its searches for GW candidates. These analyses are based on linear filters matched to $\delta$ - like signals. The algorithms implemented for the AURIGA filter and for the EXPLORER and NAUTILUS filter are different and have been independently developed. In both pipelines, the filtered data stream is calibrated to give the reconstructed Fourier component, $H$, of the strain waveform $h(t)$ of a short ( $\delta$ - like) GW burst at input.

TABLE I. Validated observation times for the 180 days considered in this analysis. Off-diagonal terms are the twofold coincidence times.

\begin{tabular}{lccc}
\hline \hline & AURIGA & EXPLORER & NAUTILUS \\
\hline AURIGA & $172.9 \mathrm{~d}$ & & \\
EXPLORER & $151.8 \mathrm{~d}$ & $158.0 \mathrm{~d}$ & \\
NAUTILUS & $150.2 \mathrm{~d}$ & $135.3 \mathrm{~d}$ & $155.0 \mathrm{~d}$ \\
\hline \hline
\end{tabular}

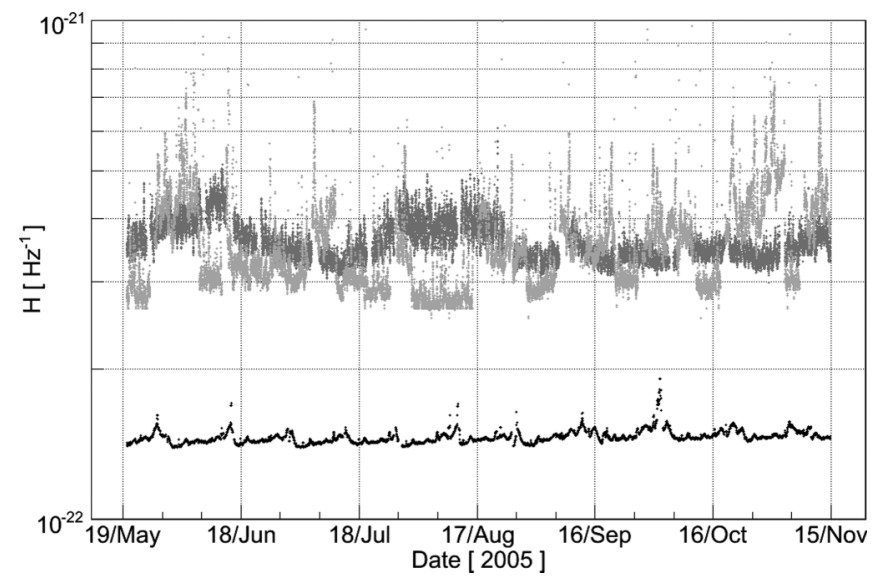

FIG. 2. Noise vs time of AURIGA (black), EXPLORER (light gray), and NAUTILUS (dark gray) detectors. The ordinate is the $1 \sigma$ noise in terms of equivalent Fourier component $H$ of the strain waveform $h(t)$ of a millisecond GW pulse.

A candidate event is identified by detecting a local maximum in the absolute value of the filtered data stream: the occurrence time of the maximum and the corresponding amplitude are the estimates of the arrival time and of the Fourier amplitude $H$ of the GW $h(t)$. Since these estimates are based on the $\delta$ filter, they are reliable only for GWs of short duration with a flat Fourier transform over the detection bandwidth. For waveforms with a colored spectrum, the filter mismatch induces a nonoptimal SNR (signal-to-noise ratio) for the candidates and a bias in their amplitude and time estimates.

As an example, in the case of signals shaped as damped sinusoids with damping time $\tau$, the typical SNR reconstructed by $\delta$ filters is $\gtrsim 80 \%$ of the SNR of the signalmatched filter for $\tau \lesssim 10 \mathrm{~ms}$, $\lesssim 25 \mathrm{~ms}$, and $\lesssim 50 \mathrm{~ms}$ for AURIGA, EXPLORER and NAUTILUS, respectively. For such waveforms, the implemented $\delta$ - filters reconstruct the arrival times with relative systematic errors $\lesssim \tau / 2$.

A cross validation has been performed on the different analysis pipelines. A sample day of raw data of EXPLORER and of NAUTILUS was processed by the AURIGA pipeline, using the same epoch vetos, but with a different implementation of the data validation and conditioning. The candidate events found by AURIGA and ROG pipelines are consistent for $\mathrm{SNR} \geq 5$ with some unavoidable differences at lower SNR.

Event lists of each detector are exchanged according to the protocol of the previous IGEC 1997-2000 observations [2], including the amplitude and time uncertainties and the amplitude threshold used to select the events.

A fixed time offset, chosen in the $\pm 10 \mathrm{~s}$ range, is added to all time information before the exchange and it is kept confidential, so that all the tuning of the analysis is performed without knowledge of the true coincidences. Once the network analysis is completely defined, these confidential time shifts are disclosed to draw the final results. This 


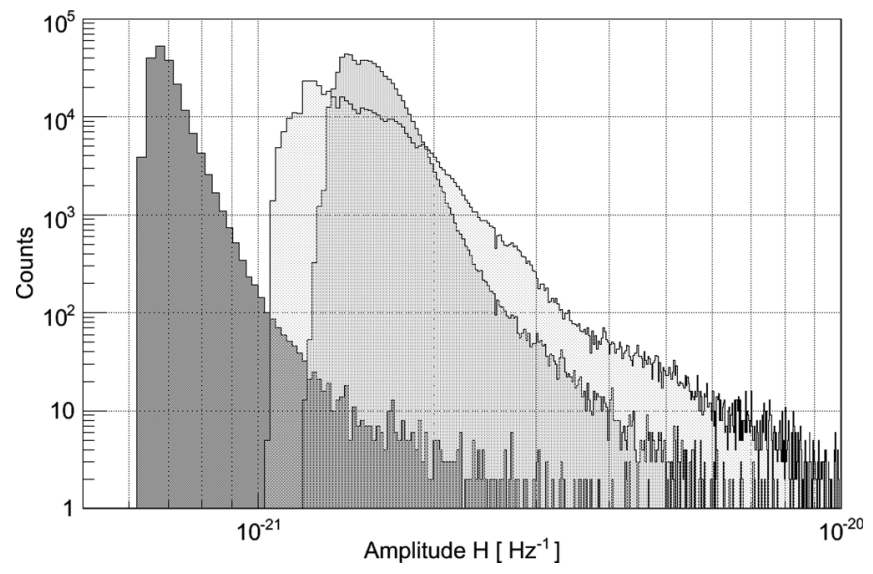

FIG. 3. Amplitude distribution of the exchanged candidate events above the minimal thresholds: AURIGA (darker gray) SNR $>4.5$, EXPLORER (lighter gray) $\mathrm{SNR}>4.0$, and NAUTILUS (gray) SNR $>4.0$. The amplitude is the Fourier component $H$ of the $h(t)$ waveform of a millisecond GW pulse.

blind analysis procedure is introduced to guarantee an unbiased statistical interpretation of the results.

The choice of exchange threshold is left to each group: $\mathrm{SNR}=4.5$ for AURIGA and $\mathrm{SNR}=4.0$ for EXPLORER and NAUTILUS. These are the minimal thresholds to identify the parameters of a single-detector candidate with reasonable confidence, as at low SNR, the timing uncertainty increases rapidly. They are tuned with hardware and software injection tests.

For the AURIGA events, the conservative estimates of the timing uncertainty $(1 \sigma)$ range from a maximum of $5 \mathrm{~ms}$ at the threshold to a minimum of $\sim 0.5 \mathrm{~ms}$ at SNR $>$ 10 , assuming $\delta-$ like signals. For EXPLORER and NAUTILUS, the $1 \sigma$ timing uncertainty is conservatively estimated to be to $10 \mathrm{~ms}$.

The amplitude distribution of the exchanged events corresponding to the period of threefold observations is shown in Fig. 3. The amplitude distribution of the AURIGA events is very close to that expected for Gaussian noise up to $\mathrm{SNR} \simeq 5.5$, while non-Gaussian outliers are dominating at higher SNRs. The number of can-

TABLE II. Number of candidate events in each detector for some SNR thresholds sets considered in the network analysis. The leftmost column refers to the minimal thresholds, the central column to the event selection optimized for signals with comparable SNR, the rightmost column to the selection optimized for signals with comparable $H$ amplitudes, see Sec. IV B.

\begin{tabular}{lccc}
\hline \hline Data cut AU & SNR $>4.5$ & SNR $>4.95$ & SNR $>7.0$ \\
EX & SNR $>4.0$ & SNR $>4.95$ & SNR $>4.25$ \\
NA & SNR $>4.0$ & SNR $>4.95$ & SNR $>4.25$ \\
\hline AURIGA & 186911 & 34598 & 790 \\
EXPLORER & 489103 & 29217 & 245000 \\
NAUTILUS & 679775 & 42028 & 351375 \\
\hline \hline
\end{tabular}

didate events above the minimal thresholds is listed in Table II; the mean event rate is $\sim 45 / h$ for AURIGA, while it is larger for EXPLORER and NAUTILUS, $\sim 129 / h$ and $\sim 183 / h$, respectively, due to the lower SNR thresholds.

\section{NETWORK DATA ANALYSIS}

The network data analysis consists of a time coincidence search among the exchanged events. The coincidence time window is set accordingly to the same procedure implemented in the IGEC 1997-2000 search [2]. Events from two detectors are in coincidence if their arrival times $t_{i}$ and $t_{j}$ are compatible within their variances, $\sigma_{i}^{2}$ and $\sigma_{j}^{2}$ :

$$
\left|t_{i}-t_{j}\right|<k \sqrt{\sigma_{i}^{2}+\sigma_{j}^{2}}
$$

where $k$ is set to 4.47, as in Ref. [2]. According to the Byenaimé-Tchebychev inequality [19], this choice limits the maximum false dismissal probability of the above coincidence condition to $5 \%$ regardless of the statistical distribution of arrival time uncertainties. For the threefold coincidence search considered here, the same condition is required per each detector pair, leading to a maximum false dismissal probability $<1-0.95^{3} \sim 14 \%$. The resulting coincidence windows are $\simeq 63 \mathrm{~ms}$ between EXPLORER and NAUTILUS and 45-50 ms when AURIGA is considered. In previous EXPLORER-NAUTILUS searches, the ROG group adopted a fixed $30 \mathrm{~ms}$ coincidence window. This value ensured a low false dismissal in the case of $\delta$-like signals considering the measured time response to excitations due to cosmic ray showers [11].

We neglected, here, the effect of the GW travel time between different sites, since it is quite small, $\leq 2.4 \mathrm{~ms}$. Moreover, if the signal duration is not small compared to the coincidence window, the quoted false dismissals are not reliable, because the systematic uncertainty on the arrival time can be different in different detectors (see the previous section).

The coincidence search is tuned to ensure a high statistical confidence in case of detection of a single gravitational wave, with an accidental rate of 1 false alarm per century. To meet this requirement, we analyzed only the 130.71 days of threefold coincident observation by AURIGA, EXPLORER and NAUTILUS. The price to be paid in sensitivity for such a low accidental rate is too high in twofold coincidence searches.

\section{A. Accidental coincidence estimates}

The threefold accidental coincidence rate was investigated with large statistics. About $20 \times 10^{6}$ independent off-source resamplings of the counting experiment were performed by shifting the data of two detectors. These shifts were within $\pm 11000 \mathrm{~s}$, in $5 \mathrm{~s}$ steps. The change in coincidence time of the resamplings is negligible, compared to the actual observation time: the average observation time of the resampled data sets is $\sim 0.09 \%$ smaller than 


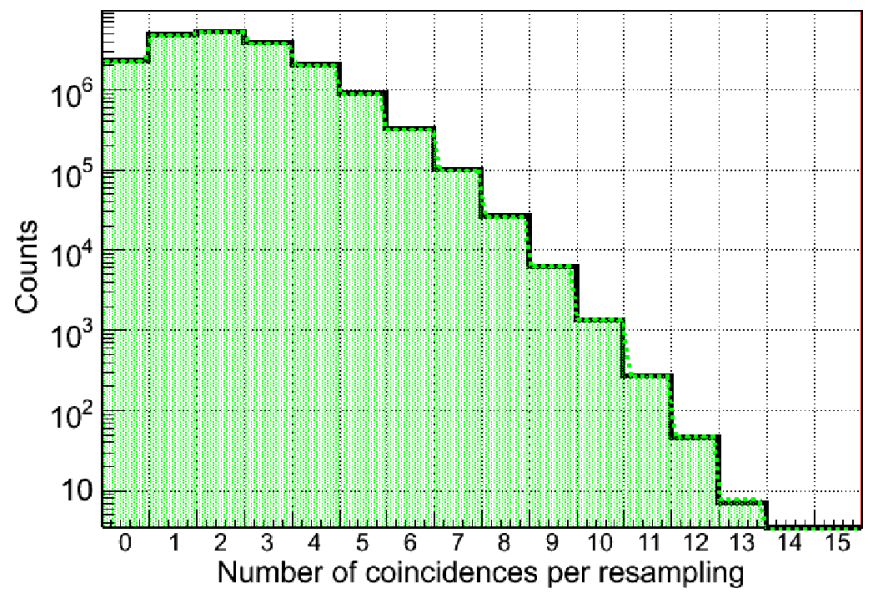

FIG. 4 (color online). Histogram of the number of accidental coincidences in each resampling (black continuous line), using exchanged events with SNR $>4.0$ for EXPLORER and NAUTILUS and SNR $>4.5$ for AURIGA. 19'355'600 independent off-source resamplings of the experiment have been computed by shifting the time of two detectors within $\pm 11000 \mathrm{~s}$ in 5 s steps, excluding the central region around the nominal zero lag (see Sec. III). The histogram is well in agreement with the Poisson distribution with mean equal to 2.16 (gray dotted line and shaded area), as $\chi^{2}=11.3$ with 12 degrees of freedom corresponding to a $p$ value of $50.3 \%$.

the actual observation time; the largest difference being $\sim 0.4 \%$. Figure 4 shows the histogram of the number of accidental coincidences in the resampled sets. The histogram is very well in agreement with a Poisson distribution of mean equal to 2.16 counts in the observation time.

This estimate was confirmed by independent coincident algorithms and different choices of the relative shifts. In all cases, the results were well within statistical fluctuations. An additional check was performed with an analytical estimate of the random coincidence rates (see appendix). The result was in very good agreement with the time-shifts technique.

\section{B. Data selection}

To achieve the goal of 1 false alarm per century, the number of accidental coincidences in the exchanged data set must be suppressed by a factor $\approx 600$, with a data selection that preserves the GW detection efficiency. In this case the balance between false alarms and detection efficiency was addressed from first principles, since measurements of average efficiency during the observation time were not available. We performed three searches, based on different data selection procedures:

(A) Fixed, equal thresholds on the event SNR in all detectors. The motivation is to set a minimal comparable event significance in all detector, as well as to have a similar rate in each detector. Because of the different spectral sensitivities, this search is most sensitive to colored signals with most of their power in the overlapping bandwidth region (e.g. 915-945 Hz, see Fig. 1). Such signals would produce similar SNRs in the $\delta$ filtered data of all detectors.

(B) Fixed thresholds on the event SNR, but different in different detectors. They are chosen to have comparable levels of absolute GW amplitude $H$ in all detectors. This search targeted short signals with flat Fourier transform in the AURIGA band, with larger SNR in AURIGA than in EXPLORER and NAUTILUS. This also allows us to use lower SNR thresholds for EXPLORER and NAUTILUS than the previous data selection procedure.

(C) Common absolute amplitude thresholds: same procedures used in the IGEC 1997-2000 search. The different data sets are selected according to a common GW amplitude $H_{i}$ [2]: the coincidence search is performed only when the exchange thresholds of all detectors are lower than $H_{i}$ and only the events whose amplitude is larger than $H_{i}$ are considered. This procedure is repeated for a grid of selected $H_{i}$ values. This search, as the previous one, targets short bursts. The main difference is that not only it selects the events, but also the effective observation time, as a function of $H_{i}$. Its main advantage is to keep under control the observatory false dismissal probability, therefore allowing an interpretation in terms of rate of GW candidates. It allows as well a straightforward comparison with the previous IGEC upper limit results.

We considered the union of these three searches, performing one composite search made by the "OR" of the three data selections procedures. This new approach simplifies the statistical analysis, since it takes care of the correlations expected in our multiple trials. In particular, the expected distribution of accidental coincidences is estimated by histogramming the union of the off-source coincidences found in the multiple trials.

Our tuning led to the following choices: A) SNR $>4.95$; B) AURIGA SNR $>7.0$, EXPLORER and NAUTILUS SNR $>4.25$, C) common search thresholds $H_{i}=$ $1.2,1.3,1.4, \ldots, 3.0 \times 10^{-21} / \mathrm{Hz}$. Table II reports the resulting number of events in each detector for data selections A) and B). In particular, with method A) the event rate is similar in all detectors, despite the larger sensitive band of AURIGA. With method B), the number of AURIGA events is a few hundred times smaller than that of EXPLORER and NAUTILUS.

The numbers of accidental coincidences found for the three data selections on the off-source resamplings are listed in Table III. Data selections B and C show a large number of common accidental coincidences, therefore their false alarms are significantly correlated.

The resulting histogram of accidental coincidences is shown in Fig. 5. The probability of a nonzero number of 
TABLE III. Number of accidental coincidences in the 19355600 off-source resamplings in each data selection method (diagonal). The off-diagonal numbers are accidentals common to different methods. Data selections B and C show a clear correlation of their accidental noises. The false alarm rates are 0.396, 0.573 , and 0.134 per century for methods $\mathrm{A}, \mathrm{B}$, and $\mathrm{C}$, respectively. The false alarm rate for the composite search, $A \cup B \cup C$ is 1.01 per century.

\begin{tabular}{lccc}
\hline \hline AU & SNR $>4.95$ & SNR $>7.0$ & Common \\
EX & SNR $>4.95$ & SNR $>4.25$ & Search \\
NA & SNR $>4.95$ & SNR $>4.25$ & Threshold \\
\hline Data cut & A & B & C \\
A & 27368 & & \\
B & 515 & 39507 & \\
C & 147 & 5177 & 9280 \\
\hline \hline
\end{tabular}

accidentals in the observation time is 0.00363 , corresponding to 1.01 false alarms per century, with $2 \times 10^{-5}$ estimated $1 \sigma$ statistical uncertainty. This uncertainty was determined by grouping the off-source samples in several disjoint subsets of equal size. The standard deviation of the number of accidentals in the subsets was propagated to the mean. The resulting $\sigma$ is only slightly higher, by a factor $\simeq 1.4$, than what is expected from a Poisson model. Independent checks with different pipelines and on different sets of off-source samples limit the systematic uncertainty on the probability to $\leqslant 1 \times 10^{-4}$.

\section{Plan of the statistical data analysis}

Before looking at the true coincidences in the on-source data set, we finalized a priori the statistical analysis plan,

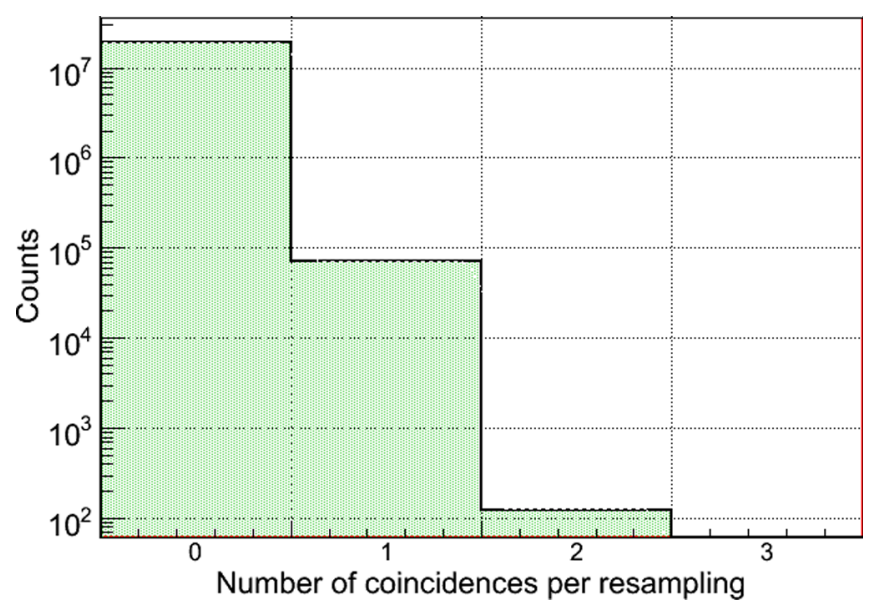

FIG. 5 (color online). Histogram of the number of accidentals in each off-source resampling from the composite search, union of three data selection procedures (see Sec. IV B). The histogram (black continuous line) agrees with a Poisson distribution with mean 0.00364 (gray dotted line and shaded area, $\chi^{2}=0.06$ with 1 degree of freedom), the reference distribution for the coincidences assuming that only accidental coincidences are present. The false detection probability is 0.00363 , corresponding to 1.01 false alarms per century. which includes two steps: the null hypothesis test and the construction of confidence intervals.

The null hypothesis is the model of the experiment which takes into account the accidental coincidences only; this hypothesis is rejected if at least one triple coincidence is found in the on-source data set. This corresponds to a $99.637 \%$ test significance, with $\pm 0.006 \% 3 \sigma$ statistical uncertainty. Therefore, if at least one coincidence is found, the Collaboration excludes it is accidental, with $99.637 \%$ confidence. The rejection of the null hypothesis would point out a correlation in the observatory, whose source may be either GWs or disturbances affecting distant detectors (e.g. instrumental correlations).

The final result on the estimated number of coincidences, related to any source of correlated noise or GWs, is given by confidence intervals ensuring a minimum coverage, i.e. the probability that the true value is included in that interval. We set confidence intervals according to the standard Feldman and Cousins confidence belt construction [20]. The noise model for the number of coincidences is the Poisson distribution shown in Fig. 5. To take into account its uncertainties, we consider the union of the confidence belts given by the mean noise $\pm 3 \sigma$, i.e. $0.00364 \pm 0.00006$ events. With such a low false alarm rate, the resulting confidence belt detaches from 0 when at least one coincidence is found, as long as the belt coverage is lower than the null hypothesis test significance. The final result cannot be easily interpreted in terms of GW source models, since IGEC-2 did not measure its detection efficiency.

Any resulting triple coincidence would then be investigated a posteriori using ALLEGRO data as well, whenever possible. These follow-up results would be interpreted in terms of likelihood, or subjective confidence, and would not affect the null hypothesis rejection significance. The goal of follow-up investigations is to discriminate among known possible sources: gravitational waves, electromagnetic or seismic disturbances, etc. More advanced network analyses, based on cross correlation would be possible with an exchange of raw data and calibration. Additional complementary information could come from electromagnetic and neutrino detectors as well as from environmental monitors.

\section{RESULTS}

Once the analysis was tuned, the groups disclosed the confidential time-shifts and looked at the actual on-source data set. This blind procedure makes the statistical interpretation of any result unambiguous. No triple coincidences were found in the composite search described in the previous sections, therefore the null hypothesis was not rejected.

The upper limit is expressed in terms of the number of detectable gravitational wave candidates, since the false dismissal of the search was not directly measured for any 
model of GW source. According to the confidence belt, the upper limits are $\simeq 2.4$ and 3.1 events at $90 \%$ and $95 \%$ coverages, respectively. For a $\mathrm{GW}$ waveform with a flat Fourier transform over the bars sensitive band, the efficiency of this search is mainly contributed by the data selection B (see Sec. IV B). In this case, according to back of the envelope calculations, IGEC-2 features a low false dismissal, $\lesssim 0.1$, at Fourier amplitudes $\gtrsim 2 \times$ $10^{-21} \mathrm{~Hz}^{-1}$ for optimally oriented sources.

For diagnostic purposes, outside the planned composite search for GWs, we also checked a posteriori the number of coincidences among all exchanged events. Three coincidences were found, well in agreement with the expected Poisson distribution of mean 2.16, discussed in Sec. IVA. The SNR of all single-detector triggers associated to these triple coincidences was close to threshold. Therefore, no additional follow-up investigation was performed.

\section{A. Comparison with IGEC previous results}

We can compute an upper limit on the rate of millisecond bursts as a function of the amplitude threshold, using the result subset relative to data cut $\mathrm{C}$. This upper limit is uninterpreted, i.e. it is set in terms of detectable GWs, as for the 1997-2000 IGEC result [2], see Fig. 6. The new upper limit improves the old one at lower amplitudes because of the better sensitivity of current detectors. The asymptotic rate, $\simeq 8.4$ events $/ \mathrm{yr}$ at $95 \%$ coverage, is higher than in the previous search because of the shorter observation time, but it is reached at much lower amplitudes. In fact, the detectors now feature more stationary performances and the current search has no false alarms, while the 1997-2000 result was dominated at low ampli-

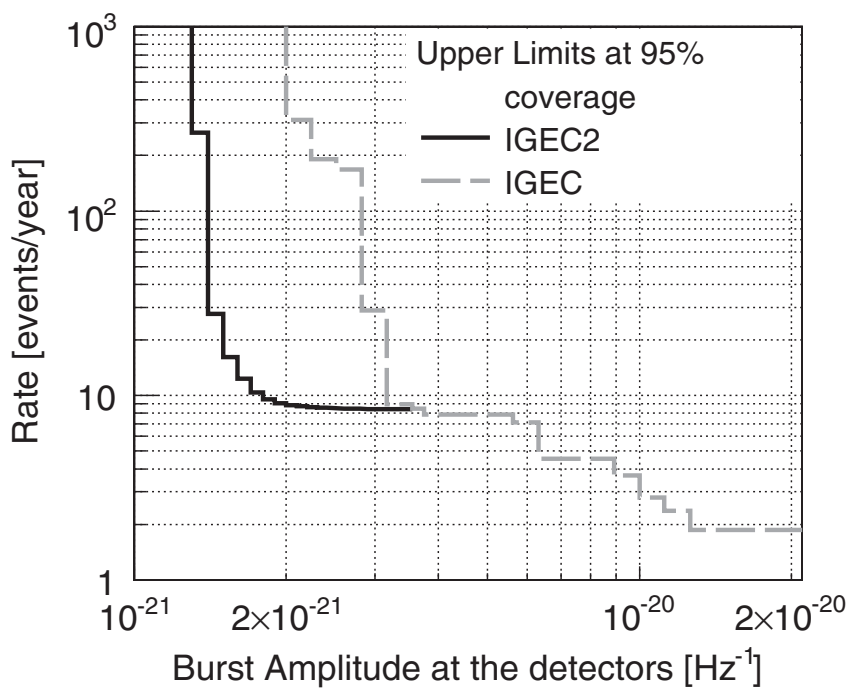

FIG. 6. Comparison between the present upper limit and the one set in the 1997-2000 campaign. These uninterpreted upper limits were computed with the same methodology. The new result, however, uses only a subset (i.e. the $\mathrm{C}$ data selection, see Sec. III) of the composite search performed on 2005 data. tudes by twofold observations with several false alarms per year.

As a general remark, the main improvement with respect to the 1997-2000 IGEC search is the low false alarm rate and the resulting ability to identify single GW candidates. In addition, the introduction of two new data selections procedures ( $\mathrm{A}$ and $\mathrm{B}$ ), extended the target of this search to a broader class of signals.

\section{FINAL REMARKS}

The IGEC-2 observatory is currently searching for GW transients. Our results show that, in a plain time coincidence analysis, a simultaneous observation by at least 3 bar detectors is necessary to identify single GW candidates with satisfactory statistical confidence. Since the LIGO spectral sensitivity in the narrow band of the bars is a factor $\sim 10$ better than the IGEC- 2 detectors, LIGO is sensitive to much weaker GW signals on a wider range of waveforms than IGEC-2 [6]. However, the resonant bar observatory can still play a significant role when the more sensitive network of interferometric detectors is unable to make a detection by itself, because not fully operational. IGEC-2 can collaborate with other observatories to extend their time coverage and contribute to the identification of rare GW events. In addition, a bar-interferometer joint investigation could increase the information on GW candidates identified by the interferometric observatory, for instance on the signal direction and polarization amplitudes. To gain the most from a hybrid bar-interferometer observatory, the analysis methods should overcome the intrinsic limitations of a plain time coincidence and exploit the phase information of the $h(t)$ data streams in different detectors, aiming at the solution of the inverse problem for the wave tensor. Tests of such methodologies are ongoing using short periods of real data sets.

\section{APPENDIX: ANALYTICAL ESTIMATE OF THE ACCIDENTAL COINCIDENCES}

The rate of accidental coincidences in the IGEC-2 observatory was empirically estimated by introducing time shifts in the data from different detectors. The result was compared to the analytical estimate described in this appendix, based on the assumption that the exchanged events are Poisson point processes with slow variable rate.

\section{Analytical model}

The expected number of accidental coincidences $N_{\text {acc }}$ in the simpler case of a constant time coincidence window $\pm w$ and constant event rate is

$$
N_{\mathrm{acc}}=M\left(\frac{w}{T_{\mathrm{obs}}}\right)^{M-1} \prod_{i=1}^{M} N_{i}
$$

where $M$ is the number of detectors, $T_{\mathrm{obs}}$ is the common 
observation time, and $N_{i}$ the number of events in the $i$ th detector.

In our case, the coincidence window $w$ depends on the detector pair $i, j$ and changes for the different AURIGA events [see Eq. (1) and Sec. III]. Therefore, the above expression for $N_{\text {acc }}$ has to be modified as shown in the following.

Given a time $t_{1}$ of an event of the detector \#1, the probability that detector \#2 has an event at a time $t_{2}$ such that $\left|t_{2}-t_{1}\right| \leq w_{12}$ is

$$
P_{12}=2 \frac{w_{12}}{T_{\mathrm{obs}}} N_{2}
$$

and similarly for detector \#3. These two probabilities are independent, so that the probability that both occur is $P_{12}$. $P_{13}$. When both occur (necessary condition for a triple coincidence) we can find the distribution of the variable $x=t_{3}-t_{2}$ by considering that the variables $t_{2}, t_{3}$ have uniform distributions in the intervals $\pm w_{12}, \pm w_{13}$ respectively. Their probability density functions (p.d.f.) are then

$$
F\left(t_{j}\right)=\frac{1}{2 w_{1 j}}
$$

with $j=2$ or 3 , and their characteristic functions (Fourier transform of the p.d.f.) are

$$
\Phi_{t_{j}}(\omega)=\frac{\sin \left(\omega w_{1 j}\right)}{\omega w_{1 j}}
$$

The characteristic function of the variable $x=t_{3}-t_{2}$ is

$$
\Phi_{x}(\omega)=\Phi_{t_{2}}(\omega) \Phi_{t_{3}}(\omega)
$$

and its p.d.f. $\mathrm{F}(\mathrm{x})$ is then given by the inverse Fourier transform of $\Phi_{x}(\omega)$

$$
F(x)=\frac{1}{2 \pi} \int_{-\infty}^{+\infty} e^{-i \omega x} \Phi_{x}(\omega) d \omega
$$

yielding the trapezoidal shape shown in Fig. 7 and described by

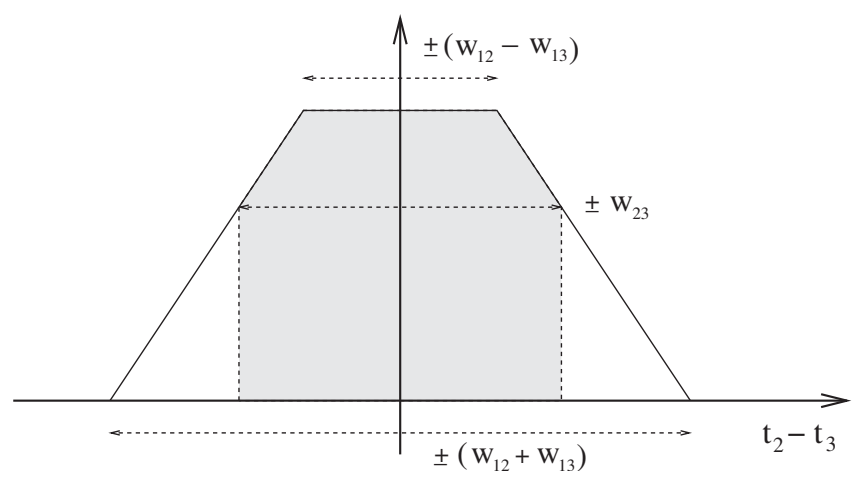

FIG. 7. P.d.f. of the variable $x=t_{2}-t_{3}$. The area of the part of the trapezium inside $\pm w_{23}$ (gray area) gives the probability of $|x| \leq w_{23}$.

$$
\begin{aligned}
F(x)= & \frac{\left|w_{12}+w_{13}+x\right|+\left|w_{12}+w_{13}-x\right|}{8 w_{12} w_{13}} \\
& -\frac{\left|w_{12}-w_{13}+x\right|+\left|w_{12}-w_{13}-x\right|}{8 w_{12} w_{13}}
\end{aligned}
$$

where we have assumed $w_{12} \geq w_{13}$.

The probability $P_{23}$ that also detectors \#2 and \#3 are in coincidence, i.e. $\left|t_{2}-t_{3}\right| \leq w_{23}$, is a fraction of the area of this trapezium:

$$
\begin{aligned}
& P_{23}=\frac{w_{23}}{w_{12}}, \quad \text { for } w_{23} \leq\left(w_{12}-w_{13}\right) ; \\
& P_{23}=1, \quad \text { for } w_{23} \geq\left(w_{12}+w_{13}\right)
\end{aligned}
$$

and in the intermediate range $w_{12}-w_{13}<w_{23}<w_{12}+$ $w_{13}$

$P_{23}=\frac{2\left(w_{12} w_{13}+w_{12} w_{23}+w_{13} w_{23}\right)-\left(w_{12}^{2}+w_{13}^{2}+w_{23}^{2}\right)}{4 w_{12} w_{13}}$.

The probability of a triple coincidence at each event of the detector \#1 is given by the product $P_{12} \cdot P_{13} \cdot P_{23}$. The number of accidental triple coincidences is obtained by further multiplying by $N_{1}$

$$
N_{\text {acc }}=4 P_{23} \frac{w_{12} w_{13}}{T_{\text {oet al.bs }}^{2}} N_{1} N_{2} N_{3},
$$

which turns to Eq. (A1) when all coincidence windows are equal to $w$.

In our case the coincidence window is set from the timing uncertainties $\sigma_{1,2,3}$ of the single events, according to Eq. (1). Then, it is easy to verify that $w_{i j}$ is bounded between the difference and the sum of the other two $w$ 's so that $P_{23}$ is given by Eq. (A8). The resulting analytical estimate for the accidental coincidences in case of constant event rates and different time windows is

$$
\begin{aligned}
N_{\mathrm{acc}}= & \frac{1}{T_{\mathrm{obs}}^{2}} N_{1} N_{2} N_{3}\left\{2\left(w_{12} w_{13}+w_{12} w_{23}+w_{13} w_{23}\right)\right. \\
& \left.-\left(w_{12}^{2}+w_{13}^{2}+w_{23}^{2}\right)\right\} .
\end{aligned}
$$

\section{Implementation}

The common observation time of the three detectors has been divided in short subintervals with a duration randomly chosen within a selected range, e.g. from $\simeq 1 / 2 \mathrm{~h}$ to $\simeq 1 \mathrm{~h}$. The minimum and maximum duration must be chosen to meet the assumptions required by Eq. (A10). In particular, the event rate should be stationary, the coincidence window much smaller than the average distance between events, and the number of accidental (background) events much larger than the number of signal (foreground) events.

Since the AURIGA events had a variable time uncertainty, we computed Eq. (A10) for each of them using 
different time windows. The prediction of $N_{\text {acc }}$ is obtained by summing the result over all the AURIGA events in the jth subinterval

$$
\begin{aligned}
N_{\mathrm{acc}}(j)= & \frac{1}{T_{j}^{2}} N_{\mathrm{Ex}}(j) N_{\mathrm{Na}}(j) \\
& \times \sum_{k=1}^{N_{\mathrm{Au}}(j)} F\left[w(E, N), w\left(A_{k}, E\right), w\left(A_{k}, N\right)\right],
\end{aligned}
$$

where $T_{j}$ is the interval duration, $N_{\mathrm{Ex} / \mathrm{Na} / \mathrm{Au}}$ are the number of events of Ex, $\mathrm{Na}, \mathrm{Au}, w(E, N)$ is the Ex-Na (fixed) coincidence window, $w\left(A_{k}, E / N\right)$ is the coincidence window $\mathrm{Au}-(\mathrm{Ex}$ or $\mathrm{Na})$ computed with the $\sigma_{t}$ of the $k$ th Auriga event, and $F(w, w, w)$ is the combination of windows in Eq. (A10). The total result for the whole overlapping period is then obtained summing over all the subintervals.

This procedure can be repeated with a different choice of the minimum and maximum intervals duration and/or with a different random initialization, in order to evaluate the fluctuations in the numerical value of the final result.
[1] Z. Allen et al., Phys. Rev. Lett. 85, 5046 (2000).

[2] P. Astone et al., Phys. Rev. D 68, 022001 (2003).

[3] P. Astone et al., Classical Quantum Gravity 18, 243 (2001).

[4] P. Astone et al., Classical Quantum Gravity 23, S169 (2006).

[5] B. Abbott et al., Phys. Rev. D 72, 062001 (2005).

[6] B. Abbott et al., Classical Quantum Gravity 24, 5343 (2007).

[7] See, for instance, http://www.ligo.caltech.edu/ and the observation paper page available at http://www.ligo.org/.

[8] M.P. McHugh, W. W. Johnson, W. O. Hamilton, J. Hanson, I. S. Heng, D. McNeese, P. Miller, D. Nettles, J. Weaver, and P. Zhang, Classical Quantum Gravity 22, S965 (2005).

[9] J. Zendri et al., Classical Quantum Gravity 19, S1925 (2002).

[10] A. Vinante et al. (AURIGA Collaboration), Classical Quantum Gravity 23, S103 (2006).

[11] P. Astone et al. (ROG Collaboration), Classical Quantum
Gravity 21, S1585 (2004).

[12] P. Astone et al., Classical Quantum Gravity 23, S57 (2006).

[13] M. Campanelli, C. Lousto, and Y. Zlochower, Phys. Rev. D 73, 061501 (2006).

[14] C. Ott, A. Burrows, L. Dessart, and E. Livne, Phys. Rev. Lett. 96, 201102 (2006).

[15] P. Astone et al., Phys. Rev. Lett. 91, 111101 (2003).

[16] M. Bignotto, M. Bonaldi, M. Cerdonio, L. Conti, F. Penasa, G. Prodi, G. Soranzo, L. Taffarello, and J. Zendri, Rev. Sci. Instrum. 76, 084502 (2005).

[17] A. Vinante, R. Mezzena, G. A. Prodi, S. Vitale, M. Cerdonio, P. Falferi, and M. Bonaldi, Appl. Phys. Lett. 79, 2597 (2001).

[18] L. Baggio et al., Phys. Rev. Lett. 94, 241101 (2005).

[19] A. Papoulis, Probability, Random Variables and Stochastic Processes (McGraw Hill, New York, 1984), 2nd ed., p. 115.

[20] G. Feldman and R. Cousins, Phys. Rev. D 57, 3873 (1998). 